Independent Submission

Request for Comments: 5831

Category: Informational

ISSN : 2070-1721
V. Dolmatov, Ed. Cryptocom, Ltd. March 2010

\title{
GOST R 34.11-94: Hash Function Algorithm
}

Abstract

This document is intended to be a source of information about the Russian Federal standard hash function (GOST R 34.11-94), which is one of the Russian cryptographic standard algorithms (called GOST algorithms). Recently, Russian cryptography is being used in Internet applications, and this document has been created as information for developers and users of GOST R 34.11-94 for hash computation.

Status of This Memo

This document is not an Internet Standards Track specification; it is published for informational purposes.

This is a contribution to the RFC Series, independently of any other RFC stream. The RFC Editor has chosen to publish this document at its discretion and makes no statement about its value for implementation or deployment. Documents approved for publication by the RFC Editor are not a candidate for any level of Internet Standard; see Section 2 of RFC 5741.

Information about the current status of this document, any errata, and how to provide feedback on it may be obtained at http://www.rfc-editor.org/info/rfc5831. 
Copyright Notice

Copyright (c) 2010 IETF Trust and the persons identified as the document authors. All rights reserved.

This document is subject to BCP 78 and the IETF Trust's Legal Provisions Relating to IETF Documents

(http://trustee.ietf.org/license-info) in effect on the date of publication of this document. Please review these documents carefully, as they describe your rights and restrictions with respect to this document.

This document may not be modified, and derivative works of it may not be created, except to format it for publication as an RFC or to translate it into languages other than English.

Table of Contents

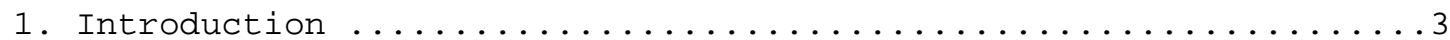

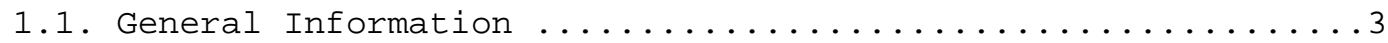

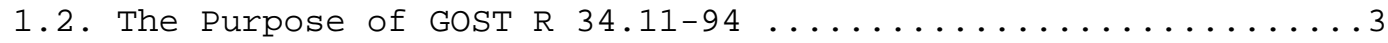

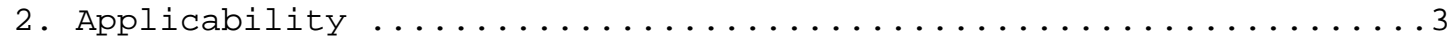

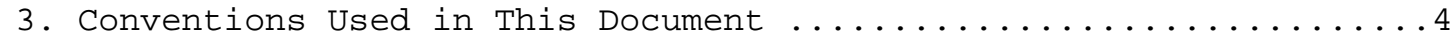

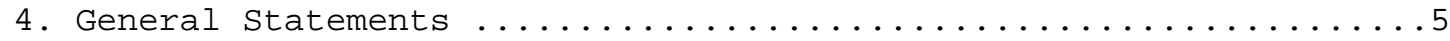

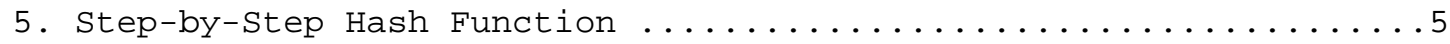

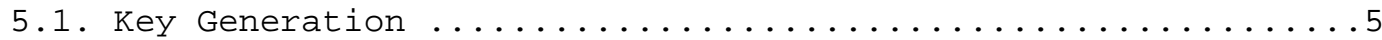

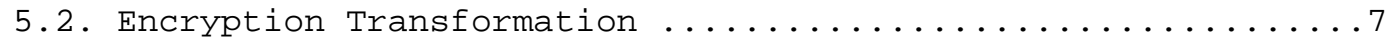

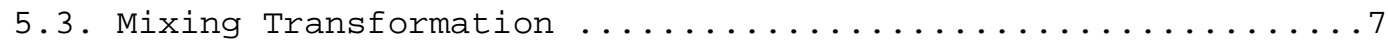

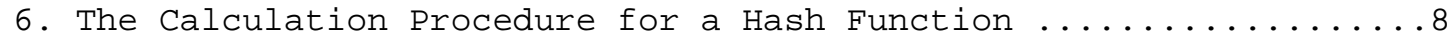

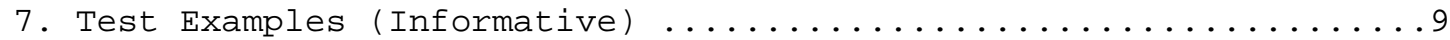

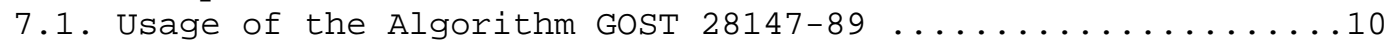

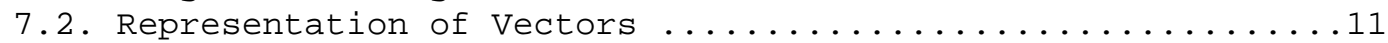

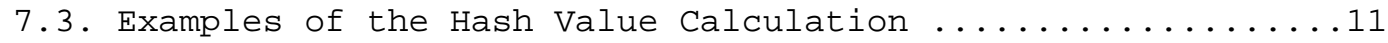
7.3.1. Hash Calculation for the Sample Message M ........11

7.3.2. Hash Calculation for the Sample Message M ........14

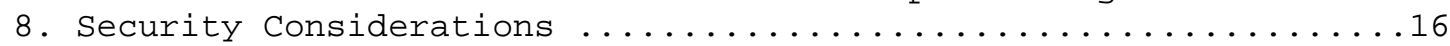

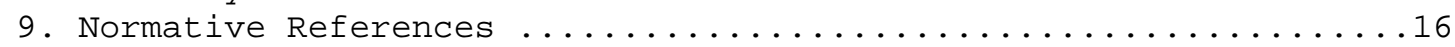

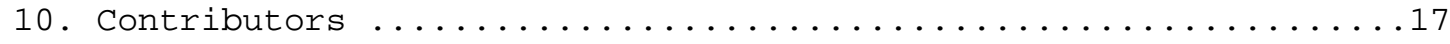




\section{Introduction}

\subsection{General Information}

1. GOST R 34.11-94 [GOST3411] was developed by the Federal Agency for Government Communication and Information and by the All-Russia Scientific and Research Institute of Standardization.

2. GOST R 34.11-94 was accepted and activated by Act 154 of 23.05.1994 issued by the Russian Federal committee for standards.

1.2. The Purpose of GOST R 34.11-94

Expanding the application of information technologies when creating, processing, and storing documents requires, in some cases, confidentiality of their contents, maintenance of completeness, and authenticity.

Cryptography (cryptographic security) is one of the effective approaches for data security. It is widely applied in different areas of government and commercial activity.

Cryptographic data security methods are under serious scientific research and standardization efforts at national, regional, and international levels.

GOST R 34.11-94 defines a hash function calculation procedure for an arbitrary sequence of binary symbols.

The hash function maps an arbitrary set of data represented as a sequence of binary symbols onto its image of a fixed small length.

Thus, hash functions can be used in procedures related to the electronic digital signature, resulting in considerable reduction of elapsed time for the sign and verify stages. The effect of the reduction of time is due to the fact that only a short image of initial data is actually signed.

\section{Applicability}

GOST R 34.11-94 defines an algorithm and procedure for the calculation of a hash function for an arbitrary sequence of binary symbols. These algorithms and procedures should be applied in cryptographic methods of data processing and securing, including digital signature procedures employed for data transfer and data storage in computer-aided systems. 
The hash function, defined in GOST R 34.11-94, is used for digital signature systems based on the asymmetric cryptographic algorithm according to GOST R 34.10-2001 (see section 3).

3. Conventions Used in This Document

The following notations are used in GOST R 34.11-94:

V_all is a set of all finite words in the alphabet $V=\{0,1\}$. The words are read from right to left and the alphabet symbols are numbered from right to left (i.e., the rightmost symbol of the word has the number one, the second rightmost symbol has number two, etc.).

$\mathrm{Vk}$ is a set of all words in alphabet $\mathrm{V}=\{0,1\}$ of length $\mathrm{k}$ bits $(\mathrm{k}=16,64,256)$.

$|A|$ is the length of a word A belonging to V_all.

$\mathrm{A}|| \mathrm{B}$ is a concatenation of words A, B belonging to V_all. Its length is $|A|+|B|$, where the left $|A|$ symbols come from the word $A$, and the right $|B|$ symbols come from the word $B$. One can also use the notation $A|| B=A$ * $B$.

$A^{\wedge} k$ is a concatenation of $k$ copies of the word $A$ ( $A$ belongs to V_all).

$\langle\mathrm{N}\rangle$ _k is a word of length $\mathrm{k}$, containing a binary representation of $\mathrm{N}\left(\bmod 2^{\wedge} \mathrm{k}\right)$ residue, with a non-negative integer $\mathrm{N}$.

$\mathrm{A} \wedge^{\wedge}$ is a non-negative integer with $\mathrm{A}$ as its binary representation. (xor) is the bitwise modulo 2 addition of the words of the same length.

$(+)^{\prime}$ is the addition according to the rule $A(+)^{\prime} B=\left\langle A^{\wedge} \$+B^{\wedge} \$\right\rangle \_k$, where $\mathrm{k}=|\mathrm{A}|=|\mathrm{B}|$.

$M$ is a binary sequence to be hashed, $M$ belongs to V_all. $M$ is a message in digital signature systems.

$h$ is a hash function that maps the sequence $M$ belonging to V_all onto the word $h(M)$ belonging to V_256.

$E(k, A)$ is a result of the encryption of the word A using key $K$ with the encryption algorithm according to [GOST28147] in the electronic codebook (ECB) mode ( $\mathrm{K}$ belongs to V256, A belongs to V64). 
h0 is an initial hash value.

e $:=g$ is the assignment of the value $g$ to the parameter $e$.

$\wedge$ is the power operator.

$i=1 . .8$ is an interval with $i$ being all the values from 1 to 8

hUZ is the S-boxes described in [GOST28147].

4. General Statements

A hash function $h$ is the mapping $h$ : V_all $\rightarrow$ V256, depending on the parameter (which is the initial hash value H, H is a word from V256). To define the hash function, it is necessary to have:

- a calculation algorithm for the step-by-step hash function chi : V256 x V256 -> V256

- a description of an iterative procedure for calculating the hash value $h$

A hash function $h$ depends on two parameters, ho and hUz.

5. Step-by-Step Hash Function

A calculation algorithm for the step-by-step hash function contains three parts, which successively do:

- key generation, here keys are 256-bit words;

- an encryption transformation, that is encryption of 64-bit subwords of word $\mathrm{H}$ using keys $\mathrm{K}[\mathrm{i}]$, ( $i=1,2,3,4$ ) with the algorithm according to [GOST28147] in ECB mode; and

- a mixing transformation for the result of the encryption.

5.1 Key Generation

Consider $\mathrm{x}=(\mathrm{b}[256], \mathrm{b}[255], \ldots, \mathrm{b}[1])$ belongs to V256.

Let :

$$
\begin{aligned}
\mathrm{x} & =\mathrm{x}[4]|| \mathrm{x}[3]|| \mathrm{x}[2]|| \mathrm{x}[1]=\operatorname{eta}[16]||[\text { eta15] }|| \ldots|| \text { eta[1] } \\
& =\mathrm{xi}[32]|| \mathrm{xi}[31]|| \ldots|| x i[1], \text { where }
\end{aligned}
$$




$$
\begin{aligned}
x[i] & =(b[i * 64], \ldots, b[(i-1) * 64+1]) \text { belongs to V64, } i=1 \ldots 4, \\
\text { eta }[j] & =(b[j * 16], \ldots, b[(j-1) * 16+1]) \text { belongs to V16, j=1..16, } \\
\text { xi }[k] & =(b[k * 8], \ldots, b[(k-1) * 8+1]) \text { belongs to V8, } k=1 \ldots 32 .
\end{aligned}
$$

Yet, another notation: $A(X)=(x[1](x \circ r) x[2])|| x[4]|| x[3]|| x[2]$.

The transformation $\mathrm{P}$ : V256 -> V256 maps the word xi32||..||xi1 onto the word xi[phi(32)] | ... ||xi[phi(1)],

where phi $(i+1+4(k-1))=8 i+k, i=0 . .3, k=1 \ldots 8$.

For the key generation, one should use the following initial data:

- words H, M belonging to V256,

- parameters: words $C[i](i=2,3,4)$, with values:

$$
\begin{aligned}
& C[2]=C[4]=0 \wedge 256 ;
\end{aligned}
$$

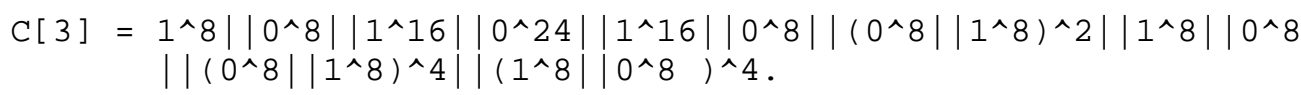

The following algorithm is used for the key calculation:

1. Assign values:

$\mathrm{i}:=1, \mathrm{U}:=\mathrm{H}, \mathrm{V}:=\mathrm{M}$.

2. Calculate:

$$
\mathrm{W}=\mathrm{U}(\mathrm{x} \circ \mathrm{r}) \mathrm{V}, \mathrm{K}[\mathrm{i}]=\mathrm{P}(\mathrm{W}) .
$$

3. Assign:

$i:=i+1$.

4. Verify condition:

$i=5$.

If it is true, go to step 7. If not, go to step 5.

5. Calculate:

$\mathrm{U}:=\mathrm{A}(\mathrm{U})(\mathrm{x} O \mathrm{r}) \mathrm{C}[\mathrm{i}], \mathrm{V}:=\mathrm{A}(\mathrm{A}(\mathrm{V}))$,

$\mathrm{W}:=\mathrm{U}(\mathrm{x} \circ \mathrm{r}) \mathrm{V}, \mathrm{K}[\mathrm{i}]=\mathrm{P}(\mathrm{W})$. 
6. Go to step 3 .

7. End.

\subsection{Encryption Transformation}

At this stage, 64-bit subwords of the word $\mathrm{H}$ are encrypted using keys K [i] $(i=1,2,3,4)$.

For the encryption transformation, one should use the following initial data:

$$
\mathrm{H}=\mathrm{h}[4]|| \mathrm{h}[3]|| \mathrm{h}[2]|| \mathrm{h}[1],
$$

where $\mathrm{h}[\mathrm{i}]$ belongs to $\mathrm{V} 64, \mathrm{i}=1,2,3,4$, and a key set is $\mathrm{K}[1], \mathrm{K}[2]$, $\mathrm{K}[3], \mathrm{K}[4]$.

The encryption algorithm is applied and the following words are obtained:

$$
\mathrm{s}[\mathrm{i}]=\mathrm{E}(\mathrm{K}[\mathrm{i}], \mathrm{h}[\mathrm{i}]) \text {, where: } i=1,2,3,4
$$

As a result of the stage, the following sequence is formed:

$$
S=s[4]|| s[3]|| s[2]|| s[1] \text {. }
$$

\subsection{Mixing Transformation}

At this stage, the obtained sequence is mixed using a shift register. The initial data includes words $\mathrm{H}, \mathrm{M}$ belonging to V256 and a word S belonging to V256.

Let a mapping PSI(X) : V256(2) -> V256(2) transform the word: eta[16]||eta[15]||...||eta[1], eta[i] belongs to V16, $i=1 \ldots 16$

into the word:

eta [1] (xor)eta [2] (xor) eta [3] (xor)eta [4] (xor)eta [13] (xor) eta [16] | |eta[16] ||...||eta[2].

Then, the value of the step-by-step hash function value is the word: $\operatorname{chi}(\mathrm{M}, \mathrm{H})=\mathrm{PSI}^{\wedge} 61\left(\mathrm{H}(\operatorname{xor}) \operatorname{PSI}\left(\mathrm{M}(\operatorname{xor}) \mathrm{PSI}^{\wedge} 12(\mathrm{~S})\right)\right)$, where PSI^i(X) is the transformation PSI applied $i$ times to $X$. 
6. The Calculation Procedure for a Hash Function

The calculation procedure for a hash function $h$ is assumed to be applied to a sequence $\mathrm{M}$ belonging to $\mathrm{V}$-all. Its parameter is an initial hash value h0, which is an arbitrarily fixed word from V256.

The calculation procedure for the function h uses the following quantities at each step of iteration:

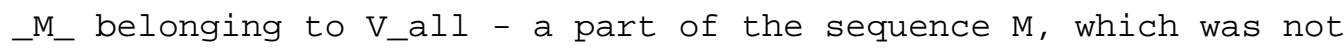
hashed at previous iterations;

H belonging to V256 - the current hash value;

SIGMA belonging to V256 - the current check sum value;

L belonging to V256 - the length of the partial sequence M processed at the previous iteration step.

The calculation algorithm for function h consists of the following steps:

Step 1. Assign initial values to current quantities:

$1.1 \_\mathrm{M}-:=\mathrm{M}$.

$1.2 \mathrm{H}:=\mathrm{h} O$.

1.3 SIGMA $:=0 \wedge 256$.

$1.4 \mathrm{~L}:=0^{\wedge} 256$.

1.5 Go to step 2 .

Step 2 .

2.1 Verify the condition $\left|{ }_{-} M_{-}\right|>256$.

If it is true, go to step 3.

Else, make the following calculations:

$2.2 \mathrm{~L}:=\left\langle\mathrm{L}^{\wedge} \$+|\mathrm{M}|>\_256\right.$

$2.3 \mathrm{M}^{\prime} \quad:=0^{\wedge}(256-|\mathrm{M}|)|| \mathrm{M}$

2.4 SIGMA $:=$ SIGMA $(+)^{\prime} \mathrm{M}^{\prime}$ 
$2.5 \mathrm{H}:=\operatorname{chi}\left(\mathrm{M}^{\prime}, \mathrm{H}\right)$

$2.6 \mathrm{H}:=\operatorname{chi}(\mathrm{L}, \mathrm{H})$

$2.7 \mathrm{H}:=\operatorname{chi}(\mathrm{SIGMA}, \mathrm{H})$

2.8 End.

Step 3 .

3.1 Calculate a subword M_s belonging to V256 of the word_M (_M_ = M_p || M_s). Then make the following calculations:

$3.2 \mathrm{H}:=$ chi (M_s, H)

$3.3 \mathrm{~L}:=\left\langle\mathrm{L}^{\wedge} \$+256\right\rangle_{-2} 256$

3.4 SIGMA $:=$ SIGMA $(+)^{\prime}$ M[S]

$3.5 \_$M_ $=$M_p

3.6 Go to step 2 .

The quantity $\mathrm{H}$ obtained at step 2.7 is the value of the hash function $\mathrm{h}(\mathrm{M})$.

7. Test Examples (Informative)

It is recommended to use the values for substitution units pi[1], pi[2],..., pi[8] and the initial hash value $H$ described in this appendix for the GOST R 34.11-94 test examples only. 
7.1. Usage of the Algorithm GOST 28147-89

The algorithm GOST 28147-89 [GOST28147] in ECB mode is used as an encryption transformation in the following examples. The following values of the substitution units pi[1], pi[2],..., pi[8] have been chosen:

$$
\begin{aligned}
& \begin{array}{llllllll}
8 & 7 & 6 & 5 & 4 & 3 & 2 & 1
\end{array} \\
& \begin{array}{lllllllll}
0 & 1 & \mathrm{D} & 4 & 6 & 7 & 5 & \mathrm{E} & 4
\end{array}
\end{aligned}
$$

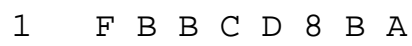

$$
\begin{aligned}
& \begin{array}{lllllllll}
2 & \mathrm{D} & 4 & \mathrm{~A} & 7 & \mathrm{~A} & 1 & 4 & 9
\end{array} \\
& \begin{array}{lllllllll}
3 & 0 & 1 & 0 & 1 & 1 & \mathrm{D} & \mathrm{C} & 2
\end{array} \\
& \begin{array}{lllllllll}
4 & 5 & 3 & 7 & 5 & 0 & \mathrm{~A} & 6 & \mathrm{D}
\end{array} \\
& \begin{array}{lllllllll}
5 & 7 & \mathrm{~F} & 2 & \mathrm{~F} & 8 & 3 & \mathrm{D} & 8
\end{array} \\
& \begin{array}{lllllllll}
6 & \mathrm{~A} & 5 & 1 & \mathrm{D} & 9 & 4 & \mathrm{~F} & 0
\end{array} \\
& \begin{array}{lllllllll}
7 & 4 & 9 & \mathrm{D} & 8 & \mathrm{~F} & 2 & \mathrm{~A} & \mathrm{E}
\end{array} \\
& \begin{array}{lllllllll}
8 & 9 & 0 & 3 & 4 & E & E & 2 & 6
\end{array} \\
& 92 \mathrm{~A} 6 \mathrm{~A} 4 \mathrm{~F} 3 \mathrm{~B} \\
& \begin{array}{lllllllll}
10 & 3 & \mathrm{E} & 8 & 9 & 6 & \mathrm{C} & 8 & 1
\end{array} \\
& \begin{array}{lllllllll}
11 & \mathrm{E} & 7 & 5 & \mathrm{E} & \mathrm{C} & 7 & 1 & \mathrm{C}
\end{array} \\
& \begin{array}{lllllllll}
12 & 6 & 6 & 9 & 0 & \mathrm{~B} & 6 & 0 & 7
\end{array} \\
& \begin{array}{lllllllll}
13 & \text { B } & 8 & \text { C } & 3 & 2 & 0 & 7 & F
\end{array} \\
& \begin{array}{lllllllll}
14 & 8 & 2 & \mathrm{~F} & \mathrm{~B} & 5 & 9 & 5 & 5
\end{array}
\end{aligned}
$$

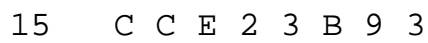

The hexadecimal value of pi[j](i) is given in a column number $j$,

$$
j=1 \ldots 8 \text {, and in a row number } i, i=0 \ldots 15 \text {. }
$$




\subsection{Representation of Vectors}

We will put down binary symbol sequences as hexadecimal digits strings, where each digit corresponds to four signs of its binary representation.

7.3 Examples of the Hash Value Calculation

A zero vector, for example, can be taken as an initial hash value:

h0 $=00000000000000000000000000000000$ $0000000000000000 \quad 00000000 \quad 00000000$

7.3.1. Hash Calculation for the Sample Message M

$M=7365747962203233 \quad 3 \mathrm{D} 687467 \quad 6 \mathrm{E} 656 \mathrm{C} 20$ $2 \mathrm{C} 656761 \quad 7373656 \mathrm{D} \quad 20736920 \quad 73696854$

Initial values are assigned for the text:

_M_ = $\quad 7365747962203233 \quad 3 \mathrm{D} 687467 \quad 6 \mathrm{E} 656 \mathrm{C} 20$ $2 \mathrm{C} 656761 \quad 7373656 \mathrm{D} 20736920 \quad 73696854$

for the hash function:

$\mathrm{H}=00000000 \quad 00000000 \quad 00000000 \quad 00000000$ $00000000 \quad 00000000 \quad 0000000000000000$

for the sum of text blocks:

SIGMA $=00000000000000000000000000000000$ $00000000 \quad 000000000000000000000000$

for the length of the text:

$L=00000000000000000000000000000000$ $00000000 \quad 00000000 \quad 00000000 \quad 00000000$

If the length of the message to be hashed equals 256 bits (32 bytes), then:

$L=00000000000000000000000000000000$ 00000000000000000000000000000100

$\mathrm{M}^{\prime}=\mathrm{M}_{-}=7365747962203233 \quad 3 \mathrm{D} 687467 \quad 6 \mathrm{E} 656 \mathrm{C} 20$ $2 \mathrm{C} 6567617373656 \mathrm{D} 20736920 \quad 73696854$ 


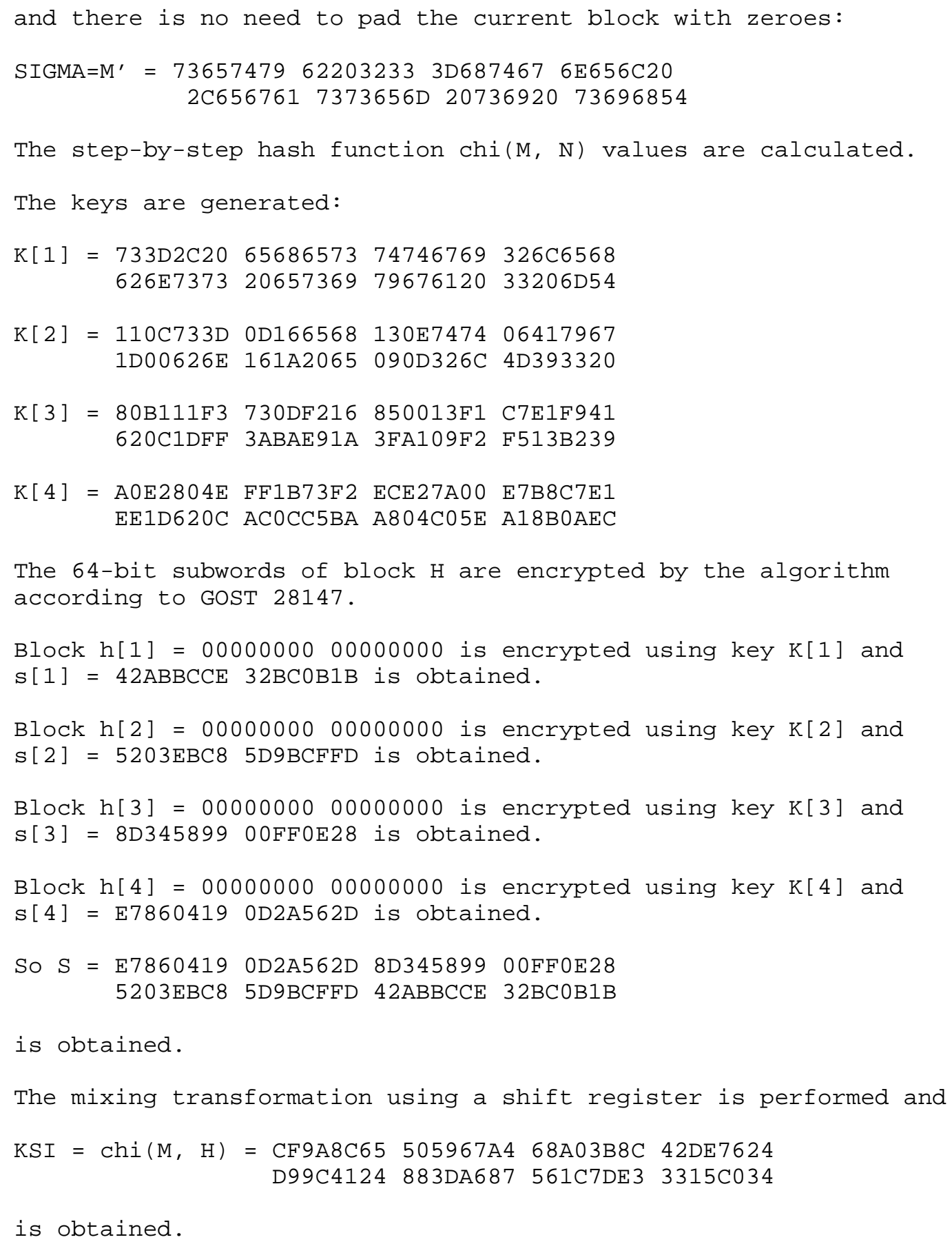




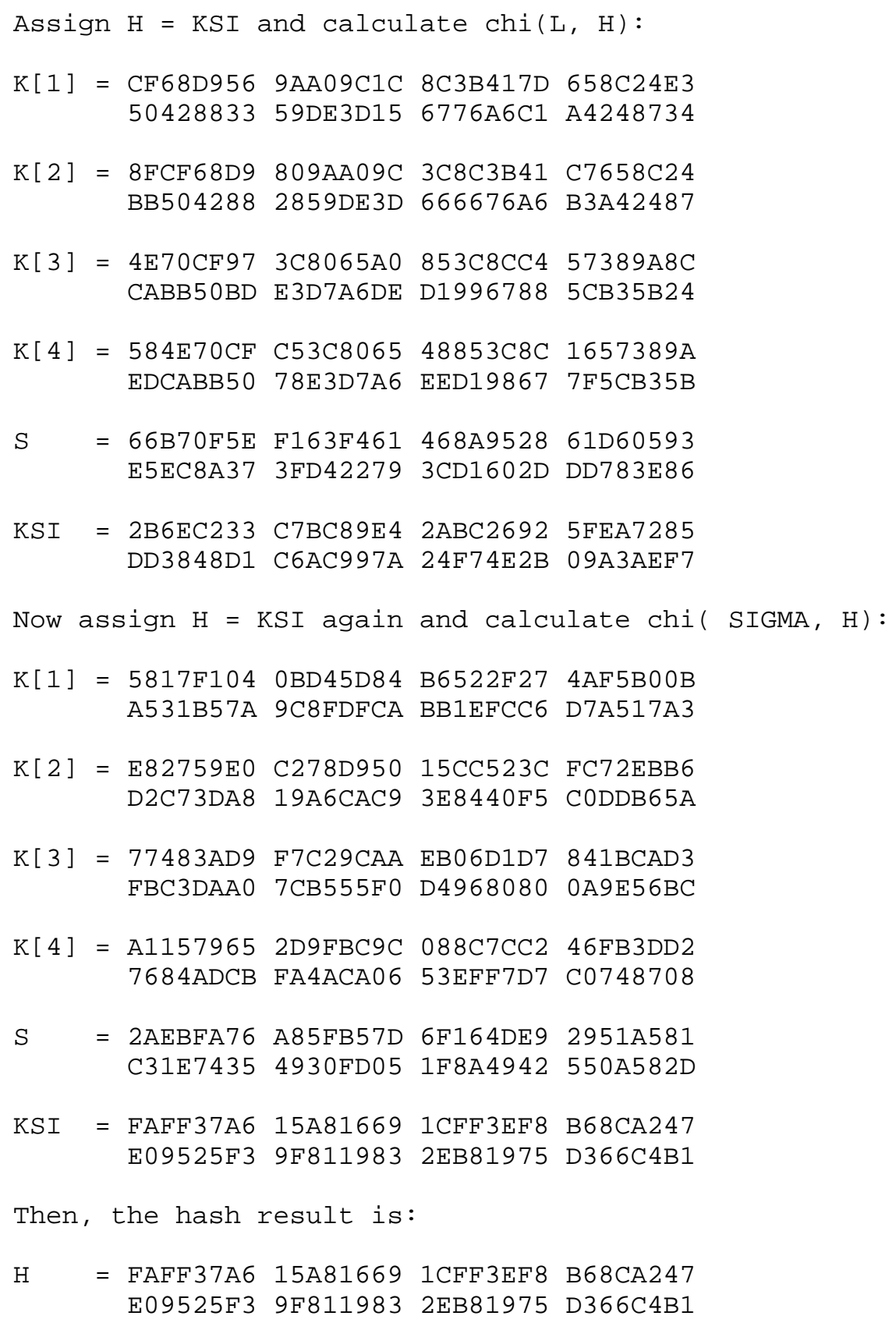




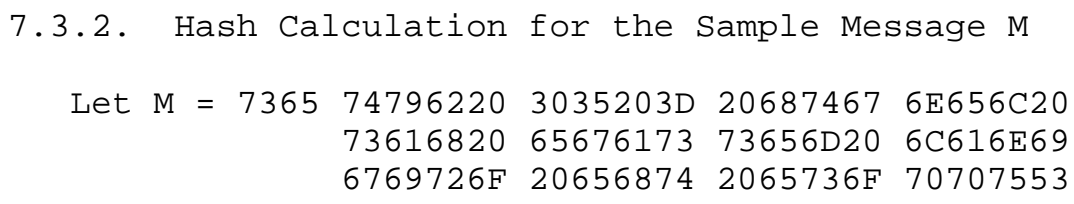




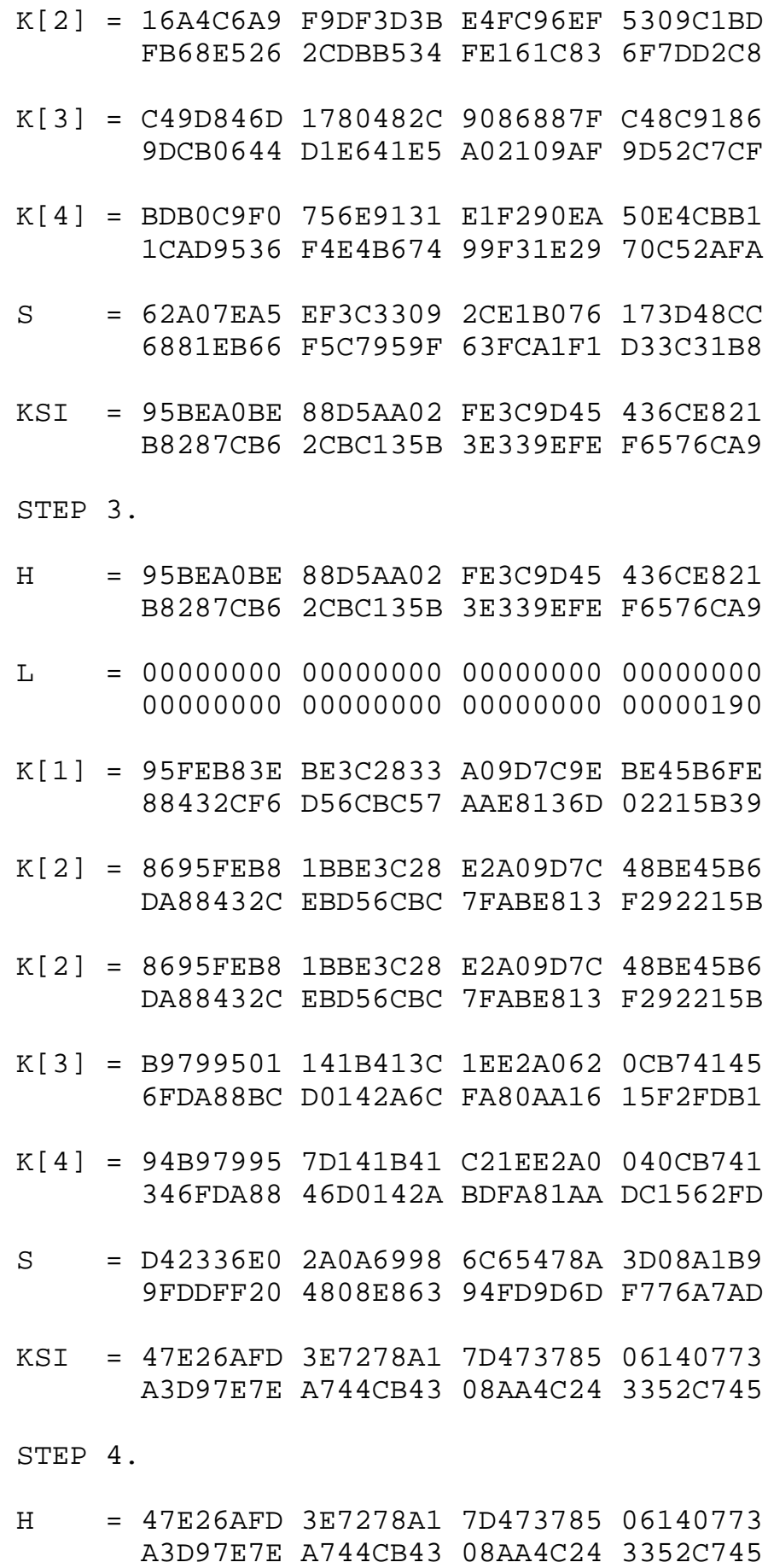




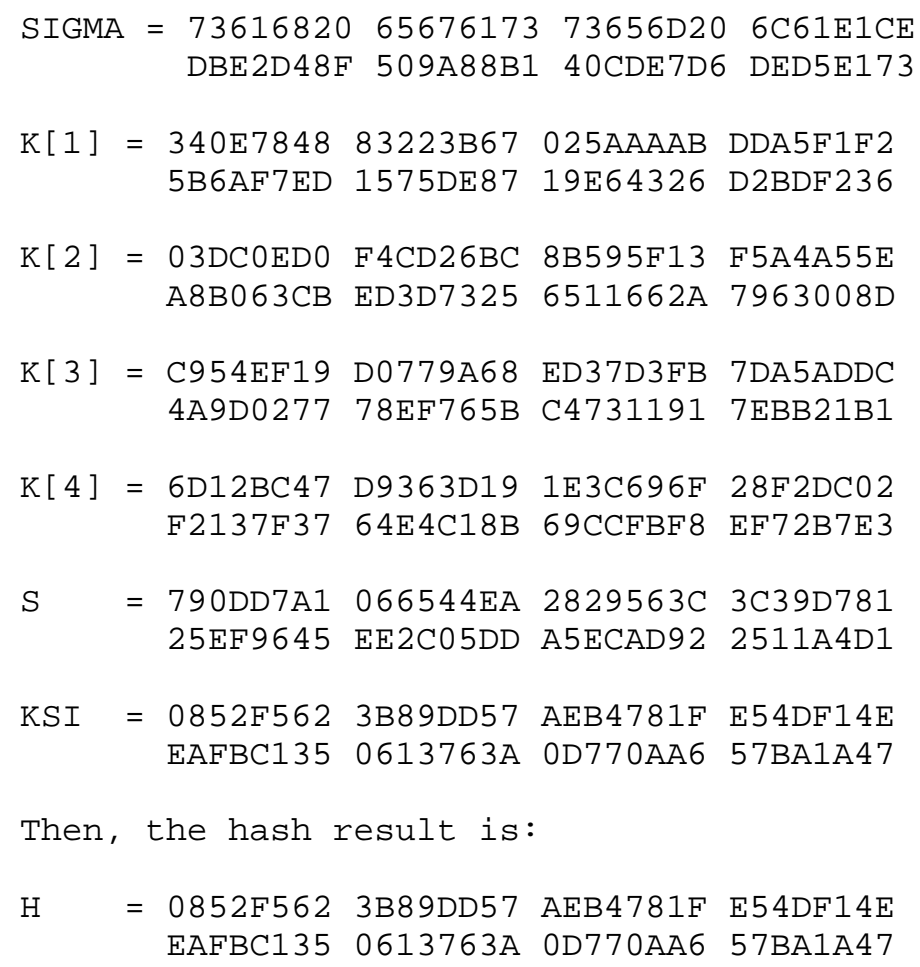

8. Security Considerations

This entire document is about security considerations.

Current cryptographic resistance of GOST R 34.11-94 hash algorithm is estimated as $2^{\wedge} 128$ operations of computations of step hash functions. (There is a known method to reduce this estimate to $2^{\wedge} 105$ operations, but it demands padding the colliding message with 1024 random bit blocks each of 256-bit length; thus, it cannot be used in any practical implementation).

9. Normative References

[GOST28147] "Cryptographic Protection for Data Processing System", GOST 28147-89, Gosudarstvennyi Standard of USSR, Government Committee of the USSR for Standards, 1989. (In Russian)

[GOST3411] "Information technology. Cryptographic Data Security. Hashing function.", GOST R 34.10-94, Gosudarstvennyi Standard of Russian Federation, Government Committee of the Russia for Standards, 1994. (In Russian) 
10. Contributors

Dmitry Kabelev Cryptocom, Ltd.

14 Kedrova St., Bldg. 2 Moscow, 117218

Russian Federation

EMail: kdbecryptocom.ru

Igor Ustinov

Cryptocom, Ltd.

14 Kedrova St., Bldg. 2

Moscow, 117218

Russian Federation

EMail: igus@cryptocom.ru

Sergey Vyshensky

Moscow State University

Leninskie gory, 1

Moscow, 119991

Russian Federation

EMail: svysh@pn.sinp.msu.ru

Author's Address

Vasily Dolmatov, Ed.

Cryptocom, Ltd.

14 Kedrova St., Bldg. 2

Moscow, 117218

Russian Federation

EMail: dolecryptocom.ru 